\title{
Complete chloroplast genome of the genus Cymbidium: lights into the species identification, phylogenetic implications and population genetic analyses
}

Jun-Bo Yang ${ }^{1}$, Min Tang ${ }^{2}$, Hong-Tao Li ${ }^{1^{*}}$, Zhi-Rong Zhang ${ }^{1}$ and De-Zhu Li $i^{*}$

\begin{abstract}
Background: Cymbidium orchids, including some 50 species, are the famous flowers, and they possess high commercial value in the floricultural industry. Furthermore, the values of different orchids are great differences. However, species identification is very difficult. To a certain degree, chloroplast DNA sequence data are a versatile tool for species identification and phylogenetic implications in plants. Different chloroplast loci have been utilized for evaluating phylogenetic relationships at each classification level among plant species, including at the interspecies and intraspecies levels. However, there is no evidence that a short sequence can distinguish all plant species from each other in order to infer phylogenetic relationships. Molecular markers derived from the complete chloroplast genome can provide effective tools for species identification and phylogenetic resolution.
\end{abstract}

Results: The complete nucleotide sequences of eight individuals from a total of five Cymbidium species' chloroplast (ср) genomes were determined using Illumina sequencing technology of the total DNA via a combination of de novo and reference-guided assembly. The length of the Cymbidium cp genome is about $155 \mathrm{~kb}$. The cp genomes contain 123 unique genes, and the IR regions contain 24 duplicates. Although the genomes, including genome structure, gene order and orientation, are similar to those of other orchids, they are not evolutionarily conservative. The cp genome of Cymbidium evolved moderately with more than 3\% sequence divergence, which could provide enough information for phylogeny. Rapidly evolving chloroplast genome regions were identified and 11 new divergence hotspot regions were disclosed for further phylogenetic study and species identification in Orchidaceae.

Conclusions: Phylogenomic analyses were conducted using 10 complete chloroplast genomes from seven orchid species. These data accurately identified the individuals and established the phylogenetic relationships between the species. The results reveal that phylogenomics based on organelle genome sequencing lights the species identification-organelle-scale "barcodes", and is also an effective approach for studying whole populations and phylogenetic characteristics of Cymbidium.

Keywords: Chloroplast genome, Phylogenomics, Species identification, Organelle-scale barcodes, Phylogeny, Divergence hotspot

\footnotetext{
*Correspondence: lihongtao@mail.kib.ac.cn; dzl@mail.kib.ac.cn

${ }^{1}$ Germplasm Bank of Wild Species, Kunming Institute of Botany, Chinese

Academy of Sciences, Kunming, Yunnan 650201, China

Full list of author information is available at the end of the article
} 


\section{Background}

Cymbidium orchids are the best known and most widely grown of all orchids. The genus Cymbidium of the orchid family Orchidaceae, consisting of 52-55 species and divided into three subgenera (Cymbidium, Cyperorchis and Jensoa) [1,2], is one of the most well-known and desirable orchids in worldwide horticulture because of its aesthetic appeal and ideal characteristics as a house plant. It is distributed in tropical and subtropical Asia and northern Australia, and species diversity centers are located in NE India, SW China, Indo-China and Malay Archipelago [1-4]. Cymbidium orchids were among the earliest to be cultivated, especially in China. Although the Cymbidium orchid species is not all widely cultivated, hybrids of Cymbidium orchids lend themselves to cultivation. Some commercially important hybrids have been produced for over one hundred years. They make excellent pot plants and cut flowers, which are the most important and popular orchids in commerce.

Because of their ornamental and commercial value, Cymbidium orchids have been the subject of taxonomic studies and, particularly, species identification [5-7]. However, so far, there are no efficient methods for identifying the species and cultivars of Cymbidium. Traditionally, the taxonomy, species and cultivars identification of the genus Cymbidium is based on the morphological traits. However, the assessment of those traits is very difficult, and morphology is affected by environmental factors. Consequently, some species are very difficult to distinguish, and the positions of many species in the evolution and taxonomy of Cymbidium are difficult to identify. Owning to widespread artificial hybridization, accurate identification of cultivated varieties via morphological traits is very difficult.

Molecular methods, such as molecular marker techniques, molecular phylogenetics and DNA barcoding, provide effective information for taxonomy, species identification and phylogenetics. In the past decades, the applications of diverse molecular techniques have gained increasing importance in resolving taxonomy, species identification and phylogenetic questions. Choi et al. [8] used RAPD markers to investigate the relationships of Cymbidium. Obara-Okeyo and Kako [9] identified Cymbidium cultivars using RAPD markers. Wang et al. [10] reported the cultivar identification in $C$. ensifolium using ISSR markers. Van den Berg et al. [5] used ITS and matK to elaborate the phylogenetic relationships of Cymbidium. Sharma et al. [6] assessed the phylogenetic inter-relationships of Cymbidium using ITS. Most of these studies revealed that a limited number of DNA sequences led to relatively little genetic variation within genus Cymbidium and therefore phylogenetic resolution and species identification were very difficult.
Owing to the DNA sequencing costs, species identification and molecular phylogenetic analyses were typically limited. It forced investigators to choose a limited number of DNA sequences with a small number of informative loci. In recent years, DNA sequencing costs have fallen dramatically with the rapid development of next-generation DNA sequencing technologies [11-16]. Simultaneously, genomics research rapidly developed allowing the efficient sequencing of large numbers of entire organellar genomes and nuclear genomes. This brought the benefits of affordable genome-scale data collection to phylogenetic resolution and species identification. As a result, it greatly increased phylogenetic resolution and species identification, especially in low taxonomic levels, i.e. genera, species, and populations.

Plastids are one of the essential organelles in plant cells. Molecular differences, based on the dissimilarities in complete chloroplast genome between plant species and individuals, offer a promising means of differentiation. The cp genomes in vascular plants have conserved quadripartite structure, composed of two copies of a large inverted repeat (IR) and two sections of unique DNA, which are referred to as the "large single copy regions" and "small single copy regions" (LSC and SSC, respectively) [17-20]. There are many advantages to the chloroplast genome in contrast to the nuclear genome such as haploid, maternal inheritance, single structure, gene content and genome structure high conservation [21-23]. Complete cp genome sequences have been widely used for plant identification and phylogenetic studies. Moore et al. [24] resolved the relationships among basal angiosperms using plastid genome-scale data. Jansen et al. [25] used 64 plastid genomes to resolve relationships between angiosperms. Parks et al. [26] used chloroplast genomes to increase phylogenetic resolution at low taxonomic levels. Moore et al. [27] used 83 chloroplast genomes to resolve the early diversification of eudicots. Wu et al. [22] used chloroplast genomes to evaluate identification and breeding in Oncidiinae. Nock et al. [28] discussed the plant identification using complete chloroplast genome. Acting as a single genome, it has become the universal method of providing evolutionary information for plant species identification, taxonomy and phylogenetic analysis.

Here, we present the complete nucleotide sequences of eight Cymbidium individuals using Illumina sequencing technology of total DNA. The aim of this study was to evaluate the role of the cp genome in taxonomy, species identification and phylogenetics. A phylogenetic tree including 10 complete $\mathrm{cp}$ genomes belonging to seven species was reconstructed. Our analyses of eight Cymbidium individuals provided detailed genetic data differentiating different individuals and species. This method demonstrated the utility of using complete chloroplast 
genome sequence information in species identification, taxonomy and phylogenetic resolution of Cymbidium.

\section{Results and discussions}

\section{Genome assembly and PCR-based validation}

Using the Illumina Hiseq 2000 system, eight individuals were sequenced to produce 5,703,656 to 7,009,641 paired-end reads (90 bp in average reads length). After screening these paired-end reads through alignment with reference cp genomes, 101,851 to 101,589 reads were mapped to the reference genomes, reaching over $100 \times$ coverage on average over the cp genome. After de novo and reference-guided assembly, two complete cp genomes were obtained. The other six cp genomes had four to nine gaps, which were then finished gap closure by PCR-based sequencing.

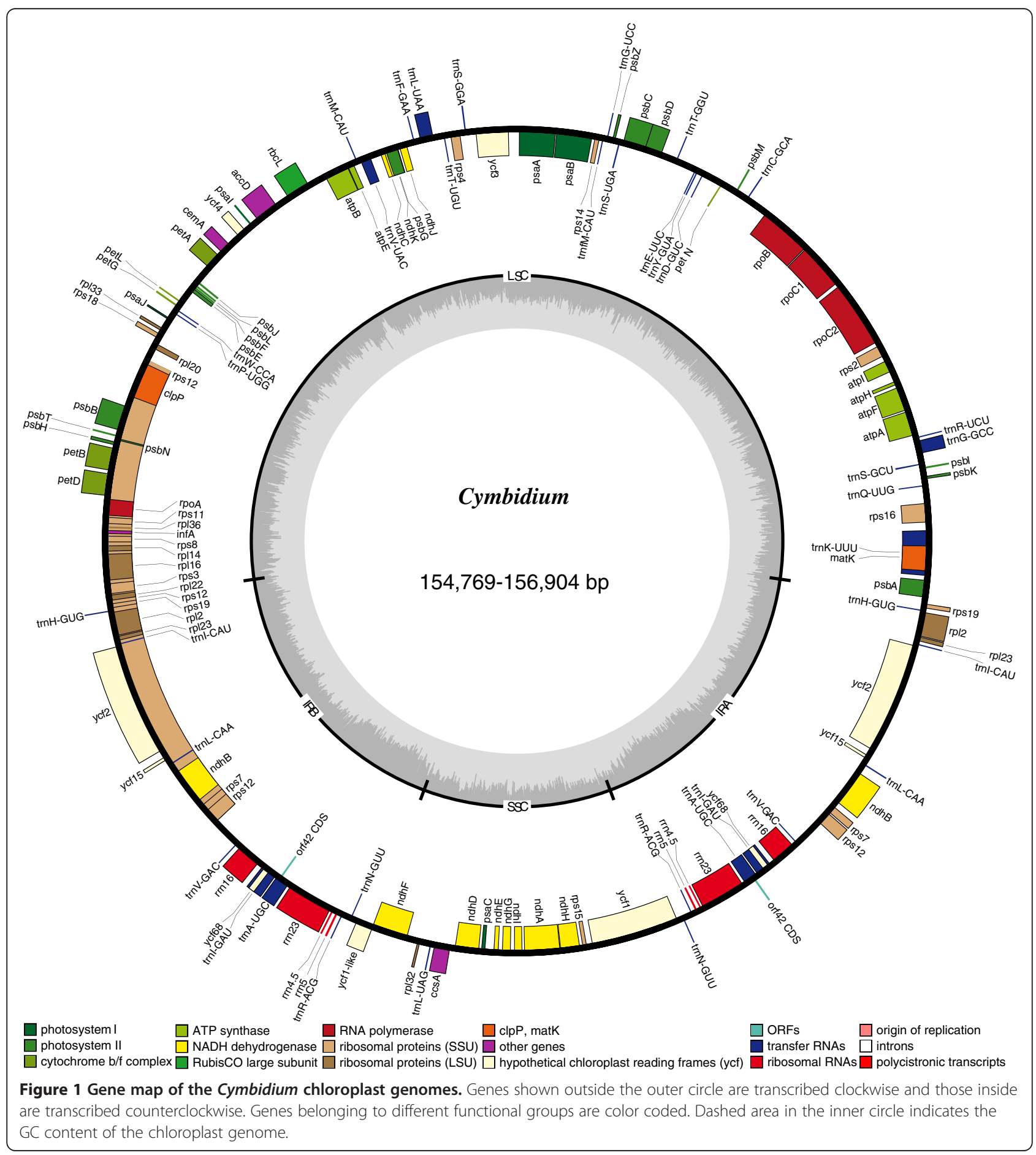


Four junction regions were validated by using PCRbased sequencing in each cp genome. Simultaneously, in order to overcome the errors of heterogeneous indels from homopolymeric repeats [16,29], we corrected the errors by PCR-based validation. We designed 62 pairs of primers based on the variation regions of alignments to validate these sequences in each cp genome (see Additional file 1: Table S1). The validated sequences from eight individuals amounted to $396,800 \mathrm{bp}$. At the same time, we had compared these sequences directly to the assembled genomes, observing no nucleotide mismatches or indels. This result validated the accuracy of our genome sequencing and assembly. We obtained complete cp genome sequences ranging from $154,769 \mathrm{bp}$ to $156,904 \mathrm{bp}$ in length.

\section{Genome features and sequence divergence}

All eight cp genomes were composed of a single the circular double-stranded DNA molecule, and they displayed the typical quadripartite structure of angiosperms, consisting of a pair of IRs (26,321-26,710 bp) separated by the LSC $(84,920-85,641 \mathrm{bp})$ and SSC (16,529-17,929 bp) regions (Figure 1). They encode an identical set of 147 predicted functional genes, of which 123 are unique and 24 are duplicated in the IR regions. The 123 unique genes are comprised of 83 proteincoding, 36 transfer RNA and 4 ribosomal RNA genes, respectively. Sixteen distinct genes, such as $\operatorname{atpF}, n d h \mathrm{~A}$, $n d h \mathrm{~B}, \operatorname{pet} \mathrm{B}, \operatorname{pet} \mathrm{D}, r p l 16, r p l 2, r p o \mathrm{C} 1, \operatorname{rps} 12, \operatorname{rps} 16, \operatorname{trn} \mathrm{A}-$ UGC, trnG-GCC, trnI-GAU, trnK-UUU, trnL-UAA and trn $\mathrm{V}$-UAC, contain one intron and two genes (clpP and $y c f 3)$ contain two introns. These introns of all proteincoding genes share the same splicing mechanism as Group II introns [30]. Some exceptional cases were identified in start codons, such as ATC for $n d h \mathrm{D}$, ACG for rpl2, ACT for rps12, GTG for rps 19 and ATT for $y c f 15$. The non-canonical start codons have been detected in other angiosperms [29,31] and tree ferns [32].

Both $y c f 15$ and $y c f 68$ genes of the IR regions became pseudogenes due to internal stop codons identified in their coding sequences (CDS). A stop codon downstream (153 bp away from the start codon) interrupts the CDS of $y c f 15$, whereas the CDS of $y c f 68$ is interrupted by two stop codons upstream (57 and 75 bp away from the start codon). Another pseudogene $y c f 1$, in the junction region of IRb and SSC exists because of incomplete duplication of the normal copy of ycf1 at the IRa and SSC junction region (Figure 1). Similar mutations occur in the cp genomes of other angiosperm species [29].

In general, $n d h$ genes widely exist in higher plants, and eleven subunits of them (ndhA-ndhK) are encoded in chloroplast genome. Yet there are exceptions: many $n d h$ genes are lacking in a number of orchids [22]. In contrast to other orchids previously studies in detail
$[22,33]$, having observed all $n d h$ gene sequences in Cymbidium, we noted that most of them are protein-coding genes except for the genes $n d h \mathrm{~A}, n d h \mathrm{D}, n d h \mathrm{~F}, n d h \mathrm{I}$, $n d h \mathrm{H}$, and $n d h \mathrm{~K}$. These $n d h$ genes could have lost their function because of abundance indels generating and stop codons existing in their CDS regions. The loss-offunction of $n d h$ genes occurs in many plants including heterotrophic and autotrophic plants [34-43]. As ndh genes have important physiological functions, we cannot explain why they lose function or are missing in the cp genome. It is possible that the functional $n d h$ genes of the cp genome have been transferred to the nuclear genome $[33,41]$, but this needs to be further explored.

Coding regions occupy $60.16 \%-60.69 \%$ of the cp genomes. $52.46 \%-53.03 \%, 1.83 \%-1.86 \%$, and $5.76 \%-5.84 \%$ of the genome sequence encodes for proteins, tRNAs, and rRNAs, respectively, whereas the remaining 39.31\%-39.84\% are non-coding regions including introns, intergenic spacers, and pseudogenes. As with other angiosperm cp genomes [31,32], the orchid cp genomes are also AT-rich and the overall AT content ranges from $63 \%$ to $63.2 \%$. The genome features of eight cp genomes-paticularly in respect to gene content, gene order, introns, intergenic spacers and AT content-are rather similar.

The cp genomes of the five Cymbidium species are distinct from most other monocots in structure and content. Usually, structural rearrangements and gene lossand gain events are quite common among monocot $\mathrm{cp}$ genomes. As a typical example, Poaceae contains three inversions in its LSC regions, which disrupt the canonical order of the cp quadripartite structure and result in the translocation of rpl23 from IR to LSC regions [44]. Indels are also common in Poaceae cp genomes such as intron-loss in rpoC1 and insertion in rpoC2 [45]. Geneloss (deletion or production of pseudogenes), particularly of the genes $a c c \mathrm{D}, y c f 1$, and $y c f 2$, are also frequently found in Poaceae cp genomes [46]. In addition, similar events also occur in other monocot families. For instance, Lemna, Dioscorea and two Acoraceae members, each lost a single gene infA, rps16 and $\operatorname{accD}[45,47,48]$, respectively, and most $n d h$ genes were lost in Phalaenopsis and Oncidium [22,33]. Beyond that, rearrangements have also been observed in monocots such as the inversion of the SSC region in Dioscorea [48]. However, similar to that of standard angiosperm cp genomes, Cymbidium cp genomes appeared less rearranged and had very little gene loss-and-gain. Recently, some groups have reported that monocot $\mathrm{cp}$ genomes have a similar structure and content to that of the Cymbidium species $[29,49]$. We observed a minor exception-an inversion at the pet $\mathrm{N}$ and $p s b \mathrm{M}$ regions.

There is moderate genetic divergence with $3.7 \%$ sequence divergence among Cymbidium species and individuals. We plotted the sequence identity using mVISTA 


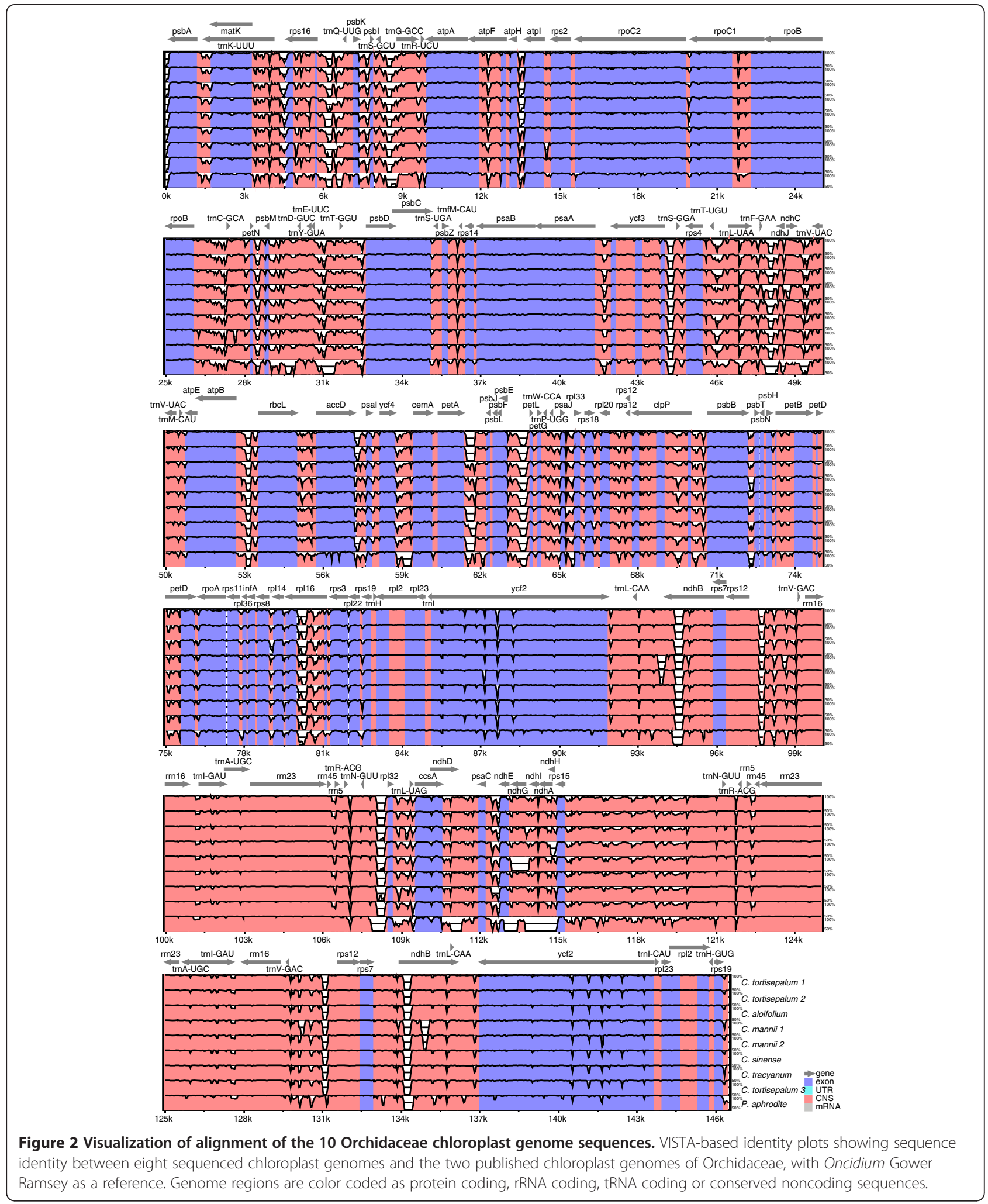

[50] by aligning the eight $\mathrm{cp}$ genomes with a reference, Oncidium Gower Ramsey (Figure 2). The whole aligned sequences reveal moderate divergences with more than 30 regions displaying below $60 \%$ identity, suggesting that orchid cp genomes have harbored rather large genetic differentiation, especially in noncoding and single copy regions (Figure 2). More than 20 divergent hotspot regions were identified (see Additional file 2: Table S2). 
The average genetic divergences of the eight Cymbidium individuals were estimated by using $p$-distance. The results showed that the $p$-distance in all individuals, between species and within individuals was $0.009,0.011$ and 0.004, respectively. The results suggested that Cymbidium individuals possess moderate genetic divergences both interspecies and intraspecies and that sequence divergences interspecies were significantly more numerous than in intraspecies divergences.

\section{Repetitive sequences}

Four categories of repeats-dispersed, tandem, palindromic and gene similarity repeats-were identified using REPuter [51] and manual verification with copy size $30 \mathrm{bp}$ or longer and sequence identity greater than 90\%. Similar to other angiosperm species [52-54], the number of repeats in Cymbidium is rather high. In all, 232 repeats were detected in eight Cymbidium cp genomes. Numbers and distributions of the four repeat types are similar and conserved among the eight $\mathrm{cp}$ genomes. Among these repeats, tandem repeats are the most common accounting for $40 \%$ of the total repeats, whereas the gene similarity repeats only occupy $5 \%$. Though a minority of repeats are found in genes such as infA, rpoC2, rps 18 and $r p s 3$, the majority are located in noncoding regions. The lengths of repeats in Cymbidium are much shorter ranging from 30 to $61 \mathrm{bp}$, whereas much longer repeats such as 91-bp and 132-bp repeats were found in the Poaceae family $[53,55]$. Previous work suggests that repeat sequences play a role in sequence rearranging and variation production in $\mathrm{cp}$ genomes through illegitimate recombination and slipped-strand mispairing [56-58]. Our research also showed that divergent regions of $\mathrm{cp}$ the genome were associated with various repeat sequences of genes such as the rpoC2 gene. Particularly, the pet $\mathrm{N}-p s b \mathrm{M}$ gene rearrangement region contained two repeat sequences. The repeat sequences might also be correlated with genome rearrangement in Cymbidium cp genomes. Furthermore, these repeats would provide valuable information for developing markers for phylogenetic research and population studies.

\section{IRs expansion}

Gene order in the four junction regions differs among various plant species. The contraction or expansion of the IR regions often results in length variation of the chloroplast genome $[18,20,31,33,59]$. This study reveals that large expansion occurred in the junction regions of Cymbidium. Around the borders of IR-LSC, Cymbidium genomes possess the typical monocot $\mathrm{cp}$ genome structure, in which the IR regions expand into the rps 19 gene region. In IRb-LSC, rpl22 and its 5'-end adjacent rps19 are completely fallen in LSC and IRb, respectively.
Similarly, in IRa-LSC, the other copy of rps19 in IRa adjoins its 3'-end to psbA in LSC. However, the borders of IR-SSC are different from typical monocots, but are similar to certain dicots in that the IR regions expand into the $y c f 1$ gene region, that is, the IRb-SSC positions itself between the $y c f 1$ pseudogene and $n d h \mathrm{~F}$. This expansion causes an overlap between the $y c f 1$ pseudogene and $n d h \mathrm{~F}$ [20], whereas IRa-SSC resides in the 3' region of the normal $y c f 1$ gene.

Among other monocots, the contraction or expansion of the IR regions were checked. Lemna, the basal monocot, had a more contracted IR regions than the basal angiosperm Amborella [25,47]. In the borders of IR-SSC, Cymbidium, the higher Poaceae and the basal Lemna had obvious expansion, whereas little expansion was checked in the other monocots $[29,49]$. It could suggest that the expansion or contraction may not be associated with phylogenetic relationship among monocots [29].

\section{Molecular marker identification}

The sequence divergence hotspot regions (>200 bp) were identified by whole cp genome-wide comparative analysis. To check the regions, which could be suitable for phylogenetic study, all of the regions were extracted from eight Cymbidium cp genomes to be used for phylogenetic analysis using the MP method. The results showed that 32 divergence hotspot regions could be subjected to Orchidaceae phylogenetic analysis. All divergence hotspot regions had a $2 \%$ composition of parsimony-informative characters. Interestingly, 11 intergenic regions (cemA-pet A, $c l p \mathrm{P}-p s b \mathrm{~B}, n d h \mathrm{~F}-r p l 32, p e t \mathrm{~A}-p s b \mathrm{~J}, p s b \mathrm{~A}-t r n \mathrm{~K}, \operatorname{trn\mathrm {L}}-c c s \mathrm{~A}$,

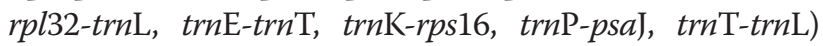
along with the commonly phylogenetic region $(\operatorname{trn} \mathrm{H}-p s b \mathrm{~A})$ had over 3\% composition of parsimony-informative characters. Compared with previous studies [5,7,60], all 11 regions harboring high phylogenetic information are newly identified in our current study.

In general, the molecular phylogenetic tree should be congruent with the evolution and life history of a species [61]. Consequently, the MP phylogenetic trees of 11 new divergence hotspot regions were constructed and were evaluated with the partition homogeneity test $[62,63]$. The results revealed that gene trees of nine new divergence hotspot regions were congruent with the combined species trees of the seven orchid species whole cp genomes (see Additional file 3: Figure S1).

In this study, we disclosed 11 new DNA variable regions harboring highly phylogenetic information, which would be potential molecular markers (see Additional file 4: Table S3) for phylogenetic study and species identification. As a result, it would be helpful if these regions were developed as markers using universal primers in order to reveal the molecular phylogeny of Orchidaceae species and species identification. 


\section{Phylogenomic analyses}

Six data partitions (complete cp DNA sequences, protein coding exons, the large single copy region, the small single copy region, inverted repeat region and the introns and spacers) from 10 orchid $\mathrm{cp}$ genomes were used to perform phylogenetic analyses. Sequence characteristics for the six datasets are shown in Additional file 5: Table S4. The small single copy region possessed the highest percentage of potential parsimony-informative characters with $3.5 \%$, and the introns and spacers with $3.2 \%$, followed by the small single copy region. The large single copy region and inverted repeat region also contained moderate genetic variation of $2.7 \%$ and $0.9 \%$ of potential parsimonyinformative characters, respectively. Furthermore, the only gene rearrangement region, $p e t \mathrm{~N}-p s b \mathrm{M}$ inversion region, is located in the LSC region. Although there were no influences on the phylogenetic tree of Cymbidium, the rearrangement region could be considered a homologous character and evolutionary marker of the genus Cymbidium that distinguishes the genus Cymbidium from other orchid genera. The protein coding exons were highly conserved and had fewer potential parsimony-informative characters less than $1.7 \%$.

Phylogenetic trees with bootstrap values (BS) and posterior probabilities (PP) were built based on the six datasets partitions (Figure 3). The method of data analyses (ML, MP and BA) had no effect on the phylogenetic trees, and the resulting topologies were highly similar in each dataset. Phylogenetic trees of the six datasets partitions were largely congruent with each
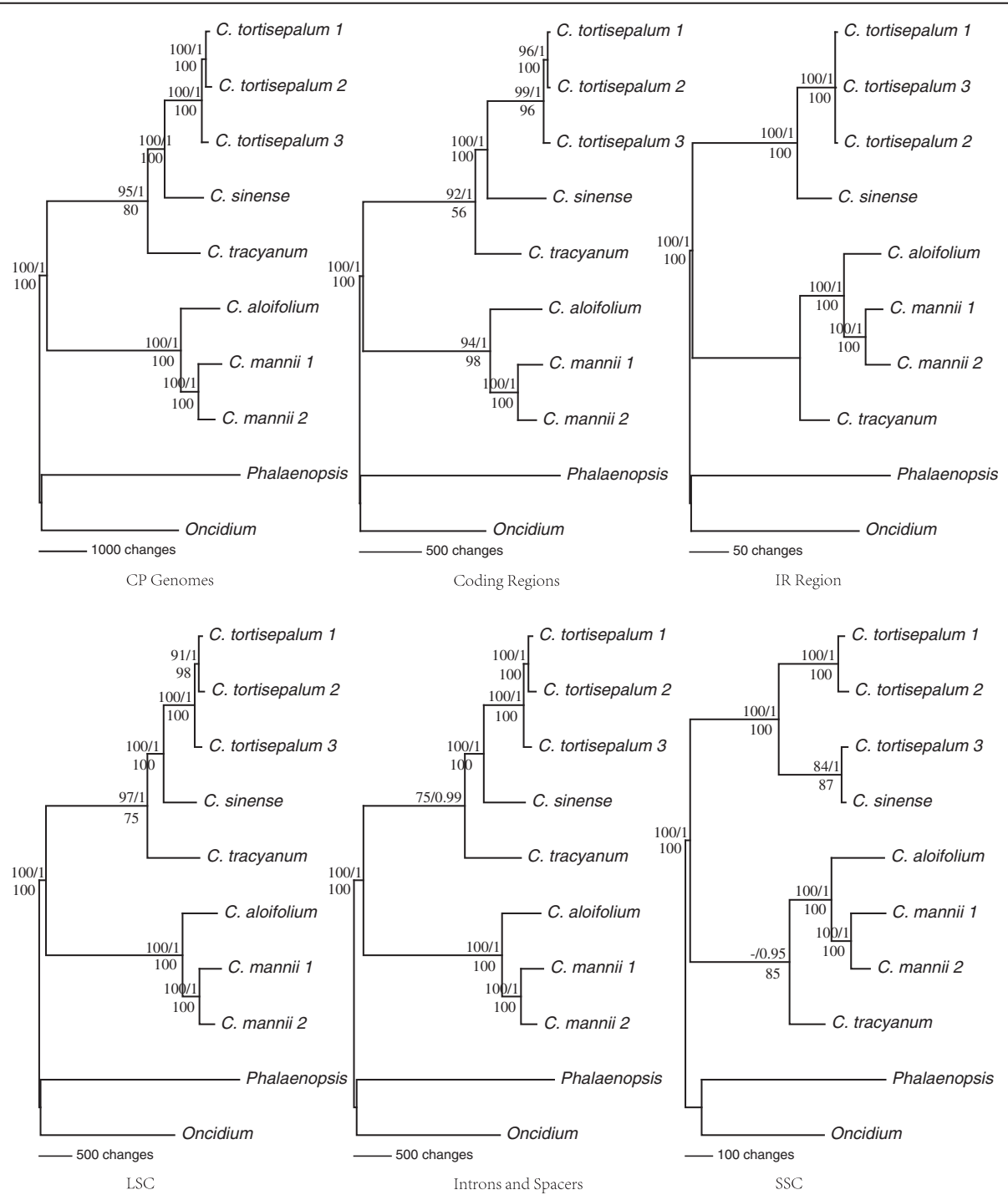

Figure 3 Maximum parsimony (MP) trees of all the six chloroplast datasets for 10 Orchidaceae individuals. Numbers above the lines on the left indicate the maximum parsimony (MP) bootstrap of each clade $>50 \%$, numbers above the lines on the right indicate the Bayesian posterior probabilities, numbers below each branch are the maximum likelihood (ML) bootstrap of each clade $>50 \%$. 
other, and only the subtle differences of topologies occurred in intraspecial clades. This suggests that there is no conflict between the partitions of cp genome. The results also revealed that the phylogenetic resolution and the support values of nodes increased significantly along with the increase of the sequence (Figure 3).

Compared with the traditional taxonomy of Cymbidium, the phylogenomic analyses also revealed that Cymbidium consists of three subgenera. However, in view of the percentage of species sampling, this needs to be verified. More importantly, different individuals of the same species from different distributions also generate moderate differentiation and can be clearly distinguished. For example, the three individuals of $C$. tortisepalum make a reliable monophyletic node, while individuals of adjacent distributions maintain sister group relationships. Additionally, the remote distribution individual maintains much divergence from other two individuals (Figure 3). Among the three individuals of $C$. tortisepalum and between the two individuals of C. mannii, there are more than 120 and 100 variable sites, respectively, which would provide enough information for cultivars identification and population-level studies. The majority of these variable sites exist in spacer

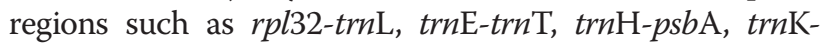
rps 16 and $\operatorname{trn} \mathrm{T}-\operatorname{trn} \mathrm{L}$. The results showed that whole cp genomes analyses could contribute to species identification, especially in cultivars identification and population-level studies. Cultivars identification plays an important role in the commercial development of orchids. Phylogenomic analyses based on whole cp genomes will light the way on cultivars identification of Cymbidium.

Compared with previous short sequence analyses in Cymbidium, our cp genomes data contained more than 100 times more parsimony-informative characters, resulting in higher-resolution nodes with much higher support values. Consequently, phylogenomic analyses based on whole cp genomes could overcome defects due to insufficient DNA sequences sampling. Our results suggest that whole cp genomes sequencing would be a feasible, reasonable and effective way for improving resolution of phylogenies, species identification and population-based studies in most land plants. Of course, considering the costs, it could reasonable be asked whether it is worth sequencing whole cp genomes for increasing species identification and phylogenetic relationship resolution. However, that is no longer an issue as a result of the rapid development of the next generation DNA sequencing technologies resulting in the sequencing costs dramatically fallen. Phylogenomics would rapidly develop based on whole cp genome analyses in the near future. However, for the rapidly radiating lineages, the whole cp genomes analyses remain insufficient to fully resolve phylogenetic relationships $[26,64,65]$ and a combined nuclear and organelle genome approach would be feasible.

\section{Conclusions}

Organelle genome sequencing is becoming a valuable way for improving resolution in phylogenetic studies with the rapid development of biotechnological sequencers, especially at low taxonomic levels. Based on the estimates of Cronn et al. [15] and the current development situation, thousands of organelle genomes could be sequenced, which would greatly mitigate current reliance on relatively short sequences in phylogenetic research [26]. It also could promote population genetic studies and species identification. Whole cp genome sequences would provide more integrated and adequate information for phylogenetic and population-based studies, improving efficient discrimination during species identification. In fact, phylogenomic studies have enjoyed recent popularity and the possibility of organelle-scale "barcodes" and population-based studies has been both considered and applied $[26,28,66]$.

Here we sequenced eight orchid individuals involving five Cymbidium species using Illumina sequencing-bysynthesis technology. These sequenced cp genomes provided genetic information on the phylogenetics, species identification and population genetic studies for these economically important orchids. These cp genomes contained moderate variations that could provide sufficient phylogenetic information for resolving evolutionary relationships. At the same time, they could also provide adequate genetic information for species identification and population genetic studies. In this study, the cp genomes accurately identified every individual and established the phylogenetic relationships between the species and individuals. The results reveal that it is an effective approach to increase the efficiency and feasibility of species identification and population-based studies while raising new questions regarding the phylogenetic implications of Cymbidium giving the characteristics of the cp genomes.

\section{Methods}

\section{Plant materials}

Five species, representing three subgenera of the genus Cymbidium, were sampled. We collected healthy, clean and fresh green leaves from adult plants of Cymbidium. The voucher herbarium specimens for the eight sampled orchids were all deposited at the Herbarium of Kunming Institute of Botany of the Chinese Academy of Sciences (KUN) (see Additional file 6: Table S5).

\section{Chloroplast DNA extraction, sequencing, genome assembly, and PCR-based validation}

Total DNA enriched for cpDNA extraction from $100 \mathrm{~g}$ fresh leaves was obtained according to the procedure outlined in Zhang et al. [17,55]. Purified DNA (5 mg) was fragmented and used to construct short-insert libraries 
according to the manufacturer's manual (Illumina). DNA from the different individuals was indexed by tags and pooled together in one lane of Illumina's Genome Analyzer for sequencing.

Since the raw sequence reads mixed non-cp DNA from the nucleus and mitochondria, we isolated the $\mathrm{cp}$ sequence reads from the raw sequence reads based on the known cp genome sequences. The filtered cp sequence reads were used to assemble the cp genomes. First, the filtered short reads were assembled into nonredundant contigs using SOAPdenovo [67], a de novo sequence assembly software, with $k=31 \mathrm{bp}$ and scaffolding contigs with a minimum size of $100 \mathrm{bp}$. Then, all contigs were mapped to the reference $\mathrm{cp}$ genomes in Orchidaceae $[22,33]$ using BLAST (http://blast.ncbi.nlm. nih.gov/) searches from NCBI with default parameters. Third, the orders of aligned contigs were determined according to the reference genomes. Finally, gaps between the de novo contigs were replaced with consensus sequences of raw reads mapped to the reference genomes.

Based on the reference genomes, the four junctions between LSC/IRs and SSC/IRs were confirmed with PCR-based product sequencing, respectively. To avoid assembly errors and to obtain high quality complete $\mathrm{cp}$ genome sequences, validation of assembly was also carried out with intensive PCR-based sequencing. We designed 62 pairs of primers based on the variation regions of the eight preliminary cp genome assemblies. PCR products were sequenced using the BigDyeV3.1 Terminator Kit for ABI 3730xl (Life Technologies). Sanger sequences and assembled genomes were aligned using Geneious [68] to determine if there were any differences. The final complete cp genome sequences were deposited into GenBank (see Additional file 6: Table S5).

\section{Genome annotation and repeat analysis}

We performed annotation of the sequenced genomes using DOGMA [69], coupled with manual corrections for start and stop codons and for intron/exon boundaries to match gene predictions from the sequenced cp genomes in Orchidaceae [22,33] of GenBank and the Chloroplast Genome Database (ChloroplastDB) http://chloro plast.cbio.psu.edu/ [70]. The sequences of identified tRNA genes were obtained using DOGMA and tRNAscan-SE (version 1.23) [71]. The functional classification of $\mathrm{cp}$ genes was referred to CpBase (http://chloroplast.ocean. washington.edu/) and ChloroplastDB. The annotated GenBank files of the cp genomes of Cymbidium were used to draw gene maps using OrganellarGenome DRAW tool (OGDRAW) [72].

Both direct and inverted repeats were assessed via REPuter [51]. Four types of repeats-dispersed, tandem, palindromic and gene similarity repeats-were observed within the Cymbidium cp genomes. The maximal length of gap size between palindromic repeats was restricted to $3 \mathrm{~kb}$. Overlapping repeats were incorporated into one repeat motif whenever possible. A given region in the genome was defined as only one repeat type, and the tandem repeat was prior to the dispersed repeat if one repeat motif could be identified as both tandem and dispersed repeats.

\section{Phylogenomic analyses}

The eight sequenced Cymbidium cp genome sequences and two public orchid cp genomes were aligned using MAFFT version 5 [73] and five loci were adjusted manually according the criteria of reducing gaps. Three ambiguously aligned loci, i.e., ' $\mathrm{N}$ ' were excluded from the analyses. The unambiguously aligned DNA sequences were used for phylogenetic and species identification analyses. To check the utilities of phylogenetic and species identification from different regions, simultaneous analyses were carried out based on the following data: (1) the complete cp DNA sequences; (2) protein coding exons; (3) the large single copy region; (4) the small single copy region; (5) the inverted repeat region; and (6) the introns and spacers. All alignments used in this study were deposited in DRYAD (accession no. doi:10.5061/dryad.14214).

Maximum likelihood (ML) and maximum parsimony (MP) analyses were conducted using PAUP $4.0 \mathrm{~b} 10$ $[74,75]$. Characters were treated as unordered and unweighted. The best model and parameter settings were chosen according to the Akaike information criterion (AIC) as suggested by Modeltest V3.7 [76,77] for the ML analyses. Heuristic searches were conducted with tree-bisection reconnection (TBR) branch swapping, MulTrees ON, and 10,000 random taxon addition replicates holding 20 trees at each step. Bootstrap support (BS) values for individual clades were calculated by running 1,000 bootstrap replicates of the data, with starting trees acquired by a single replicate of random stepwise addition of taxa under TBR branch swapping, with MulTrees ON. The consistency index (CI), retention index (RI), rescaled consistency index (RC), and pairwise distances were obtained through PAUP $4.0 \mathrm{~b} 10$ as the actual number of site differences excluded indels.

Bayesian analyses (BA) were conducted using MrBayes $3.2[78,79]$. The best model and parameters settings were chosen according to the Akaike information criterion (AIC) as suggested by ModelTest v3.7 [76,77]. The results were based on the best-fit models of the AIC test. Four independent Markov Chain Monte Carlo chains were run simultaneously and sampled every 100 generations for a total of 1,000,000 generations. To establish the "burn-in" phase, i.e., log probability values stationarity, a plot of generations against log likelihood scores 
was generated; these burn-in trees were discarded from the analysis.

\section{p-distance calculation}

To assess the utility of complete $\mathrm{cp}$ genome sequencing in species identification and population genetics, the aligned complete $\mathrm{cp}$ genome sequences were used to calculate p-distance with MEGA5 [80]. Missing data were treated as complete deletions.

\section{Molecular marker identification}

To examine the divergence regions for phylogenetic applications, all the regions, including coding regions, introns and intergenic spacers from eight Cymbidium cp genomes, were sequentially extracted. Every homologous region was aligned using MUSCLE [81] and further manual adjustments were made where necessary. As a result, the percentage of variable characters for every region was calculated.

For the divergence hotspot regions, the maximum parsimony method was used to construct the phylogenetic trees with PAUP4.0b10 to check the congruence of evolution and life history of the species. Heuristic tree searches were conducted with 10,000 random-taxon -addition replicates holding 20 trees at each step and tree bisection-reconnection (TBR) branch swapping, with the "MulTrees" option in effect. Non-parametric bootstrap analysis was conducted using 1,000 replicates with TBR branch swapping.

\section{Additional files}

Additional file 1: Table S1. Primers used for gap closure, assembly and junction verification.

Additional file 2: Table S2. Percentage of parsimony-informative characters in 32 divergence hotspot regions among eight Cymbidium individuals.

Additional file 3: Figure S1. Maximum parsimony (MP) trees of nine regions from the 11 new DNA divergence hotspot regions of 10 Orchidaceae individuals.

Additional file 4: Table S3. Primers for 11 potential molecular markers. Additional file 5: Table S4. DNA site variation and tree statistics for the six datasets used in the phylogenomic analyses presented in this study.

Additional file 6: Table S5. Sampled species and voucher specimens of Cymbidium used in this study.

\section{Competing interests}

The authors declare that they have no competing interests.

\section{Authors' contributions}

JBY, HTL and DZL designed research and wrote the paper. JBY, MT, HTL and ZRZ performed research. HTL analyzed data. All authors read and approved the final manuscript.

\section{Acknowledgments}

We are very grateful to Mr. Wu-Xiang Fu, Ms. Juan-Hong Zhang, Mr. Jia-Lin Huang and Ms. Na Yang of Kunming Institute of Botany for their help with the experiments. This work was funded by the National Natural Science
Foundation of China (31260487), the Chinese Academy of Sciences through a Large-Scale Scientific Facilities Research Project (2009-LSFGBOWS-01) and Instruments Development and Technology Innovation Project of the Chinese Academy of Sciences (Integrated Development System for Acquiring Plant Genetic Information Efficiency).

\section{Author details}

${ }^{1}$ Germplasm Bank of Wild Species, Kunming Institute of Botany, Chinese Academy of Sciences, Kunming, Yunnan 650201, China. ${ }^{2}$ College of Landscape and Horticulture, Yunnan Agricultural University, Kunming, Yunnan 650201, China.

Received: 27 December 2012 Accepted: 16 April 2013 Published: 18 April 2013

\section{References}

1. Chen XQ, Liu Z, Zhu GH, Lang KY, Ji ZH, Luo YB, Jin XH, Cribb PJ, Wood JJ, Gale SW, et al: Orchidaceae. In Flora of China. Edited by Wu ZY, Raven PH, Hong DY. Beijing \& St. Louis: Science Press \& Missouri Botanical Garden Press; 2009:260.

2. Du Puy D, Cribb P: The Genus Cymbidium, 2nd edn. Surrey, United Kingdom: Royal Botanic Gardens, Kew Publishing; 2007.

3. Du Puy D, Cribb P: The genus Cymbidium. London and Portland, Oregon: Christopher Helm and Timber Press; 1988.

4. Liu ZJ, Chen SC, Ru ZZ: The genus Cymbidium in China. Beijing, China: Science Press; 2006.

5. van den Berg C, Ryan A, Cribb PJ, Chase MW: Molecular phylogenetics of Cymbidium (Orchidaceae: Maxillarieae): Sequence data from internal transcribed spacers (ITS) of nuclear ribosomal DNA and plastid matK. Lindleyana 2002, 17(2):102-111.

6. Sharma SK, Dkhar J, Kumaria S, Tandon P, Rao SR: Assessment of phylogenetic inter-relationships in the genus Cymbidium (Orchidaceae) based on internal transcribed spacer region of rDNA. Gene 2012, 495(1):10-15.

7. Pornarong S: DNA barcoding of the Cymbidium species (Orchidaceae) in Thailand. Afr J Agric Res 2012, 7(3):393-404.

8. Choi SH, Kim MJ, Lee JS, Ryu KH: Genetic diversity and phylogenetic relationships among and within species of oriental cymbidiums based on RAPD analysis. Sci Hortic 2006, 108(1):79-85.

9. Obara-Okeyo P, Kako S: Genetic diversity and identification of Cymbidium cultivars as measured by random amplified polymorphic DNA (RAPD) markers. Euphytica 1998, 99(2):95-101.

10. Wang HZ, Lu JJ, Hu X, Liu JJ: Genetic variation and cultivar identification in Cymbidium ensifolium. Plant Syst Evol 2011, 293(1-4):101-110.

11. Hudson ME: Sequencing breakthroughs for genomic ecology and evolutionary biology. Mol Ecol Resour 2008, 8(1):3-17.

12. Mardis ER: The impact of next-generation sequencing technology on genetics. Trends Genet 2008, 24(3):133-141.

13. Shendure J, Ji H: Next-generation DNA sequencing. Nat Biotechnol 2008, 26(10):1135-1145

14. Tangphatsornruang S, Sangsrakru D, Chanprasert J, Uthaipaisanwong P, Yoocha $\mathrm{T}$, Jomchai N, Tragoonrung S: The chloroplast genome sequence of mungbean (Vigna radiata) determined by high-throughput pyrosequencing: structural organization and phylogenetic relationships. DNA Res 2010, 17(1):11-22.

15. Cronn R, Liston A, Parks M, Gernandt DS, Shen R, Mockler T: Multiplex sequencing of plant chloroplast genomes using Solexa sequencing-bysynthesis technology. Nucleic Acids Res 2008, 36(19):e122.

16. Moore MJ, Dhingra A, Soltis PS, Shaw R, Farmerie WG, Folta KM, Soltis DE: Rapid and accurate pyrosequencing of angiosperm plastid genomes. BMC Plant Biol 2006, 6:17.

17. Jansen RK, Raubeson LA, Boore JL, dePamphilis CW, Chumley TW, Haberle RC, Wyman SK, Alverson AJ, Peery R, Herman SJ, et al: Methods for obtaining and analyzing whole chloroplast genome sequences. Methods Enzymol 2005, 395:348-384.

18. Saski C, Lee SB, Daniell H, Wood TC, Tomkins J, Kim HG, Jansen RK: Complete chloroplast genome sequence of Glycine max and comparative analyses with other legume genomes. Plant Mol Biol 2005, 59(2):309-322.

19. Palmer JD, Stein DB: Conservation of chloroplast genome structure among vascular plants. Curr Genet 1986, 10(11):823-833.

20. Kim KJ, Lee HL: Complete chloroplast genome sequences from Korean ginseng (Panax schinseng Nees) and comparative analysis of sequence evolution among 17 vascular plants. DNA Res 2004, 11(4):247-261. 
21. Palmer JD, Jansen RK, Michaels HJ, Chase MW, Manhart JR: Chloroplast DNA variation and plant phylogeny. Ann Mo Bot Gard 1988, 75(4):1180-1206

22. Wu FH, Chan MT, Liao DC, Hsu CT, Lee YW, Daniell H, Duvall MR, Lin CS: Complete chloroplast genome of Oncidium Gower Ramsey and evaluation of molecular markers for identification and breeding in Oncidiinae. BMC Plant Biol 2010, 10:68.

23. Tian X, Li DZ: Application of DNA sequences in plant phylogenetic study. Acta Bot Yunnanica 2002, 24(2):170-184.

24. Moore MJ, Bell CD, Soltis PS, Soltis DE: Using plastid genome-scale data to resolve enigmatic relationships among basal angiosperms. Proc Natl Acad Sci USA 2007, 104(49):19363-19368.

25. Jansen RK, Cai Z, Raubeson LA, Daniell H, Depamphilis CW, Leebens-Mack J, Muller KF, Guisinger-Bellian M, Haberle RC, Hansen AK, et al: Analysis of 81 genes from 64 plastid genomes resolves relationships in angiosperms and identifies genome-scale evolutionary patterns. Proc Natl Acad Sci USA 2007, 104(49):19369-19374.

26. Parks $M$, Cronn R, Liston A: Increasing phylogenetic resolution at low taxonomic levels using massively parallel sequencing of chloroplast genomes. BMC Biol 2009, 7:84.

27. Moore MJ, Soltis PS, Bell CD, Burleigh JG, Soltis DE: Phylogenetic analysis of 83 plastid genes further resolves the early diversification of eudicots. Proc Natl Acad Sci USA 2010, 107(10):4623-4628.

28. Nock CJ, Waters DL, Edwards MA, Bowen SG, Rice N, Cordeiro GM, Henry RJ: Chloroplast genome sequences from total DNA for plant identification. Plant Biotechnol J 2011, 9(3):328-333.

29. Yang M, Zhang X, Liu G, Yin Y, Chen K, Yun Q, Zhao D, Al-Mssallem IS, Yu J: The complete chloroplast genome sequence of date palm (Phoenix dactylifera L.). PLoS One 2010, 5(9):e12762.

30. Sugita M, Sugiura M: Regulation of gene expression in chloroplasts of higher plants. Plant Mol Biol 1996, 32(1-2):315-326.

31. Raubeson LA, Peery R, Chumley TW, Dziubek C, Fourcade HM, Boore JL, Jansen RK: Comparative chloroplast genomics: analyses including new sequences from the angiosperms Nuphar advena and Ranunculus macranthus. BMC Genomics 2007, 8:174

32. Gao L, Yi X, Yang YX, Su YJ, Wang T: Complete chloroplast genome sequence of a tree fern Alsophila spinulosa: insights into evolutionary changes in fern chloroplast genomes. BMC Evol Biol 2009, 9:130.

33. Chang CC, Lin HC, Lin IP, Chow TY, Chen HH, Chen WH, Cheng CH, Lin CY, Liu SM, Chang CC, et al: The chloroplast genome of Phalaenopsis aphrodite (Orchidaceae): Comparative analysis of evolutionary rate with that of grasses and its phylogenetic implications. Mol Biol Evol 2006 23(2):279-291.

34. dePamphilis CW, Palmer JD: Loss of photosynthetic and chlororespiratory genes from the plastid genome of a parasitic flowering plant. Nature 1990, 348(6299):337-339.

35. Wickett NJ, Zhang Y, Hansen SK, Roper JM, Kuehl JV, Plock SA, Wolf PG, DePamphilis CW, Boore JL, Goffinet B: Functional gene losses occur with minimal size reduction in the plastid genome of the parasitic liverwort Aneura mirabilis. Mol Biol Evol 2008, 25(2):393-401.

36. Haberhausen $\mathrm{G}$, Zetsche K: Functional loss of all ndh genes in an otherwise relatively unaltered plastid genome of the holoparasitic flowering plant Cuscuta reflexa. Plant Mol Biol 1994, 24(1):217-222.

37. McNeal JR, Kuehl JV, Boore $J$, de Pamphilis CW: Complete plastid genome sequences suggest strong selection for retention of photosynthetic genes in the parasitic plant genus Cuscuta. BMC Plant Biol 2007, 7:57

38. Revill MJ, Stanley S, Hibberd JM: Plastid genome structure and loss of photosynthetic ability in the parasitic genus Cuscuta. J Exp Bot 2005, 56(419):2477-2486

39. Cameron KM: Utility of plastid psaB gene sequences for investigating intrafamilial relationships within Orchidaceae. Mol Phylogenet Evol 2004, 31(3):1157-1180

40. Cameron KM, Molina MC: Photosystem II gene sequences of $p s b B$ and $p s b C$ clarify the phylogenetic position of Vanilla (Vanilloideae, Orchidaceae). Cladistics 2006, 22(3):239-248.

41. Wakasugi T, Tsudzuki J, Ito S, Nakashima K, Tsudzuki T, Sugiura M: Loss of all ndh genes as determined by sequencing the entire chloroplast genome of the black pine Pinus thunbergii. Proc Natl Acad Sci USA 1994, 91(21):9794-9798.

42. Wu CS, Lai YT, Lin CP, Wang YN, Chaw SM: Evolution of reduced and compact chloroplast genomes (cpDNAs) in gnetophytes: selection toward a lower-cost strategy. Mol Phylogenet Evol 2009, 52(1):115-124.
43. McCoy SR, Kuehl JV, Boore $J$, Raubeson LA: The complete plastid genome sequence of Welwitschia mirabilis: an unusually compact plastome with accelerated divergence rates. BMC Evol Biol 2008, 8:130.

44. Doyle JJ, Davis Jl, Soreng RJ, Garvin D, Anderson MJ: Chloroplast DNA inversions and the origin of the grass family (Poaceae). Proc Natl Acad Sci USA 1992, 89(16):7722-7726.

45. Goremykin W, Holland B, Hirsch-Ernst Kl, Hellwig FH: Analysis of Acorus calamus chloroplast genome and its phylogenetic implications. Mol BiO Evol 2005, 22(9):1813-1822.

46. Maier RM, Neckermann $\mathrm{K}$, Igloi GL, Kossel H: Complete sequence of the maize chloroplast genome: gene content, hotspots of divergence and fine tuning of genetic information by transcript editing. J Mol Biol 1995 251(5):614-628

47. Mardanov AV, Ravin NV, Kuznetsov BB, Samigullin TH, Antonov AS, Kolganova TV, Skyabin KG: Complete sequence of the duckweed (Lemna minor) chloroplast genome: structural organization and phylogenetic relationships to other angiosperms. J Mol Evol 2008, 66(6):555-564.

48. Hansen DR, Dastidar SG, Cai Z, Penaflor C, Kuehl JV, Boore JL, Jansen RK: Phylogenetic and evolutionary implications of complete chloroplast genome sequences of four early-diverging angiosperms: Buxus (Buxaceae), Chloranthus (Chloranthaceae), Dioscorea (Dioscoreaceae), and Illicium (Schisandraceae). Mol Phylogenet Evol 2007, 45(2):547-563.

49. Guisinger MM, Chumley TW, Kuehl JV, Boore JL, Jansen RK: Implications of the plastid genome sequence of Typha (Typhaceae, Poales) for understanding genome evolution in Poaceae. J Mol Evol 2010, 70:149-166.

50. Mayor C, Brudno M, Schwartz JR, Poliakov A, Rubin EM, Frazer KA, Pachter LS, Dubchak I: VISTA : visualizing global DNA sequence alignments of arbitrary length. Bioinformatics 2000, 16(11):1046-1047.

51. Kurtz S, Choudhuri JV, Ohlebusch E, Schleiermacher C, Stoye J, Giegerich R: REPuter: the manifold applications of repeat analysis on a genomic scale. Nucleic Acids Res 2001, 29(22):4633-4642.

52. Bausher MG, Singh ND, Lee SB, Jansen RK, Daniell H: The complete chloroplast genome sequence of Citrus sinensis (L.) Osbeck var 'Ridge Pineapple': organization and phylogenetic relationships to other angiosperms. BMC Plant Biol 2006, 6:21.

53. Saski C, Lee SB, Fjellheim S, Guda C, Jansen RK, Luo H, Tomkins J, Rognli OA, Daniell H, Clarke JL: Complete chloroplast genome sequences of Hordeum vulgare, Sorghum bicolor and Agrostis stolonifera, and comparative analyses with other grass genomes. Theor App/ Genet 2007, 115(4):571-590.

54. Lee SB, Kaittanis C, Jansen RK, Hostetler JB, Tallon L, Town CD, Daniell H: The complete chloroplast genome sequence of Gossypium hirsutum: organization and phylogenetic relationships to other angiosperms. BMC Genomics 2006, 7:61.

55. Zhang YJ, Ma PF, Li DZ: High-throughput sequencing of six bamboo chloroplast genomes: phylogenetic implications for temperate woody bamboos (Poaceae: Bambusoideae). PLoS One 2011, 6(5):e20596.

56. Asano T, Tsudzuki T, Takahashi S, Shimada H, Kadowaki K: Complete nucleotide sequence of the sugarcane (Saccharum officinarum) chloroplast genome: A comparative analysis of four monocot chloroplast genomes. DNA Res 2004, 11(2):93-99.

57. Timme RE, Kuehl JV, Boore $J$, Jansen RK: A comparative analysis of the Lactuca and Helianthus (Asteraceae) plastid genomes: Identification of divergent regions and categorization of shared repeats. Am J Bot 2007, 94(3):302-312.

58. Cavalier-Smith T: Chloroplast evolution: secondary symbiogenesis and multiple losses. Curr Biol 2002, 12(2):R62-R64.

59. Chung HJ, Jung JD, Park HW, Kim JH, Cha HW, Min SR, Jeong WJ, Liu JR: The complete chloroplast genome sequences of Solanum tuberosum and comparative analysis with Solanaceae species identified the presence of a 241-bp deletion in cultivated potato chloroplast DNA sequence. Plant Cell Rep 2006, 25(12):1369-1379.

60. Freudenstein JV, van den Berg C, Goldman DH, Kores PJ, Molvray M, Chase MW: An expanded plastid DNA phylogeny of Orchidaceae and analysis of jackknife branch support strategy. Am J Bot 2004, 91(1):149-157.

61. Smith SA, Donoghue MJ: Rates of molecular evolution are linked to life history in flowering plants. Science 2008, 322(5898):86-89.

62. Farris JS, Kallersjo M, Kluge AG, Bult C: Testing significance of incongruence. Cladistics 1994, 10(3):315-319.

63. Farris JS, Kallersjo M, Kluge AG, Bult C: Constructing a significance test for incongruence. Syst Biol 1995, 44(4):570-572. 
64. Fishbein M, Hibsch-Jetter C, Soltis DE, Hufford L: Phylogeny of Saxifragales (angiosperms, eudicots): analysis of a rapid, ancient radiation. Syst Biol 2001, 50(6):817-847.

65. Wortley AH, Rudall PJ, Harris DJ, Scotland RW: How much data are needed to resolve a difficult phylogeny? Case study in Lamiales. Syst Biol 2005, 54(5):697-709.

66. Kuang DY, Wu H, Wang YL, Gao LM, Zhang SZ, Lu L: Complete chloroplast genome sequence of Magnolia kwangsiensis (Magnoliaceae): implication for DNA barcoding and population genetics. Genome 2011, 54(8):663-673.

67. Li R, Zhu H, Ruan J, Qian W, Fang X, Shi Z, Li Y, Li S, Shan G, Kristiansen K, et al: De novo assembly of human genomes with massively parallel short read sequencing. Genome Res 2010, 20(2):265-272.

68. Meintjes P, Duran C, Kearse M, Moir R, Wilson A, Stones-Havas S, Cheung M, Sturrock S, Buxton S, Cooper A, et al: Geneious Basic: An integrated and extendable desktop software platform for the organization and analysis of sequence data. Bioinformatics 2012, 28(12):1647-1649.

69. Wyman SK, Jansen RK, Boore JL: Automatic annotation of organellar genomes with DOGMA. Bioinformatics 2004, 20(17):3252-3255.

70. Cui L, Veeraraghavan N, Richter A, Wall K, Jansen RK, Leebens-Mack J, Leebens-Mack J, Makalowska I, dePamphilis CW: ChloroplastDB: the chloroplast genome database. Nucleic Acids Res 2006, 34:D692-D696.

71. Lowe TM, Eddy SR: tRNAscan-SE: a program for improved detection of transfer RNA genes in genomic sequence. Nucleic Acids Res 1997, 25(5):955-964.

72. Lohse M, Drechsel O, Bock R: OrganellarGenomeDRAW (OGDRAW): a tool for the easy generation of high-quality custom graphical maps of plastid and mitochondrial genomes. Curr Genet 2007, 52(5-6):267-274.

73. Katoh K, Kuma K, Toh H, Miyata T: MAFFT version 5: improvement in accuracy of multiple sequence alignment. Nucleic Acids Res 2005, 33(2):511-518.

74. Swofford DL: PAUP*. Phylogenetic analysis using parsimony (*and other methods). version 4. Sunderland, Massachusetts: Sinauer Associates; 2002.

75. Wilgenbusch JC, Swofford D: Inferring evolutionary trees with PAUP*, Current protocols in bioinformatics / editoral board, Andreas D Baxevanis [et al]. Malden, MA, United States: John Wiley \& Sons, Inc.; 2003. Chapter 6:Unit 6.4.

76. Posada D, Crandall KA: MODELTEST: testing the model of DNA substitution. Bioinformatics 1998, 14(9):817-818.

77. Posada D: Using MODELTEST and PAUP* to select a model of nucleotide substitution, Current protocols in bioinformatics / editoral board, Andreas D Baxevanis [et al.]. Malden, MA, United States: John Wiley \& Sons, Inc; 2003. Chapter 6:Unit 6.5.

78. Ronquist F, Teslenko M, van der Mark P, Ayres DL, Darling A, Hohna S, Larget B, Liu L, Suchard MA, Huelsenbeck JP: MrBayes 3.2: Efficient bayesian phylogenetic inference and model choice across a large model space. Syst Biol 2012, 61(3):539-542.

79. Ronquist F, Huelsenbeck JP: MrBayes 3: Bayesian phylogenetic inference under mixed models. Bioinformatics 2003, 19(12):1572-1574.

80. Tamura K, Peterson D, Peterson N, Stecher G, Nei M, Kumar S: MEGA5: Molecular evolutionary genetics analysis using maximum likelihood, evolutionary distance, and maximum parsimony methods. Mol Biol Evol 2011, 28(10):2731-2739.

81. Edgar RC: MUSCLE: multiple sequence alignment with high accuracy and high throughput. Nucleic Acids Res 2004, 32(5):1792-1797.

doi:10.1186/1471-2148-13-84

Cite this article as: Yang et al:: Complete chloroplast genome of the genus Cymbidium: lights into the species identification, phylogenetic implications and population genetic analyses. BMC Evolutionary Biology 2013 13:84.

\section{Submit your next manuscript to BioMed Central and take full advantage of:}

- Convenient online submission

- Thorough peer review

- No space constraints or color figure charges

- Immediate publication on acceptance

- Inclusion in PubMed, CAS, Scopus and Google Scholar

- Research which is freely available for redistribution 